\title{
Separation thresholds for colored bars with and without luminance contrast'
}

Separation thresholds for rectangular bar targets were obtained for certain combinations of black, white and colored targets and grounds. Relatively low threshold separation scores were obtained with colored targets against white grounds with targets and ground equated in luminance. The results suggest that color contrast is sufficient but less efficient than luminance contrast for visual acuity.

Theories of visual acuity have been mainly theories of intensity or contrast discrimination. Most investigations which have included color, or more properly, wavelength as a variable, have been concerned with questions of improving contrast or luminance gradients at the critical contours. Typically, but not unanimously, these investigators have used black targets on colored grounds and have found acuity to be best with illuminants from the yellow to green region of the visual spectrum and relatively worse with illuminants from the extremes of the spectrum.

The fact that color contrast alone may be a sufficient basis for visual acuity has been indicated by some work (e.g. Eastman Kodak Company, 1944) but most of the small amount of data available is fragmentary or gathered under special observation conditions which render evaluation and integration of the data difficult at best.

The present preliminary study with simple targets and viewing conditions is one of a series initiated with the goal of gathering more information on color contrast as a variable in visual acuity.

Subjects

One female and three males, ages 21 to 37 , served in the experiment. The three males wore corrective lenses and all Ss scored 1.0 or better in the far and near monocular visual acuity test on the AO Ortho-rater. All Ss scored $100 \%$ on the Ishihari and the AO tests for color deficiency.

Method

The stimulus configuration presented to the $\mathrm{S}$ was a pair of rectangular bars seen against a circular ground. The bars subtended a visual angle of approximately $0.5^{\circ}$ in width and $1.1^{\circ}$ in height at $63 \mathrm{~cm}$, and the ground an angle of $7^{\circ}$. A larger annular surround concentric with the ground increased the illuminated field to approximately $22^{\circ}$. The optical arrangement of the apparatus was such that light from a single $450-\mathrm{W}$ Xenon source was directed to three secondary diffuse sources for the surround, ground and targets respectively. The light from these sources was directed to the $S$ 's eye in a modified Maxwellian manner. This arrange- ment allowed independent control of the luminance and color characteristics of the display elements. The annular surround was always white with a luminance level set at the $5 \mathrm{ft}-\mathrm{L}$ level used for the ground and targets. Target and ground colors were controlled with Corning and Wratten filters. The filters used and their calculated dominant wavelengths were: C(Corning) 2-59, $640 \mathrm{~nm}$; Wratten No. 73, $571 \mathrm{~nm}$; $\mathrm{C}-4-64,528 \mathrm{~nm} ; \mathrm{C}-5-60,458 \mathrm{~nm}$.

During an experimental session the $S$ was comfortably seated before the apparatus with his eye position rigidly controlled and fixed with use of an adjustable bite board bearing his dental impression. Observation was monocular with a $3-\mathrm{mm}$ artificial pupil.

After $1 / 2 \mathrm{hr}$. dark adaptation the $\mathrm{S}$ was positioned and light adapted to white light at $5 \mathrm{ft}-\mathrm{L}$ for $5 \mathrm{~min}$. After 10 practice adjustments with black targets presented on a white ground he was then adapted to the white for 2 min. after which the first test stimulus condition was presented.

The task for the $\mathrm{S}$ was to make two sorts of adjustments of the target bar pair. With the bars well apart he brought them together to the point at which the line of separation just was not seen, a together judgment. This setting was read from a calibrated dial on the target carriage mechanism and the targets were then moved more closely together. The S then moved the targets apart until he just perceived a line of separation, an apart judgment. Ss were allowed to bracket the judgment point but were restricted to making the last movement in the indicated direction. The Ss made five pairs of judgments for each stimulus combination with a 1-min. adaptation to white light between each combination. Thresholds were obtained for all combinations during a single session, and four counterbalanced replications were obtained for each $\mathrm{S}$.

\section{Results and Discussion}

The curves in Figs. 1 and 2 show the mean separation threshold scores for all Ss combined. Each point is based on 20 pairs of judgments for each of the four Ss. The curves are representative although details differed between Ss.

In Fig. 1, curve $\mathrm{B} / \mathrm{C}$ shows the separation threshold scores obtained with black targets presented against colored grounds. The pattern of the obtained scores, low with the yellow and greater with the blue and red grounds, is consistent with acuity data gathered with use of black bars on colored grounds (Ferree \& Rand, 1931) and black grids on colored grounds (Shlaer et al, 1942). 


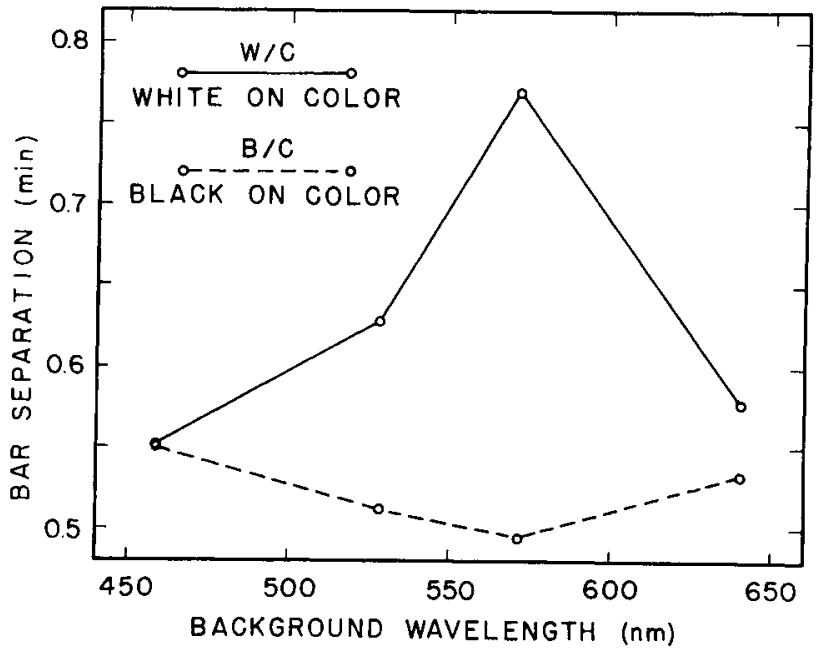

Fig. 1. Mean detectable bar separation of $0.5^{\circ} \times 1.1^{\circ}$ white and black bars presented on different colored grounds.

Curve W/C shows the data obtained with the white bars presented on colored grounds. The inverted pattern of the data, with very large separation scores for the white on yellow condition seems to reflect mainly the loss or change in color contrast between the bars and the thin colored line of separation. So called small angle tritanopia and the perceptual loss of color of very small stimuli has been discussed by many investigators (e.g. Middleton \& Holmes, 1949). Both the separation scores and subjective report of the observers in the present experiment parallel previous qualitative descriptions of the perceptual changes. Very small yellow and green stimuli appear white or light grey, red and blue appear dark grey or black; and the loss of color occurs at larger angular size with yellow and blue stimuli than with green and red.

In Fig. 2 the scores obtained with colored targets on a black ground are shown in curve $\mathrm{C} / \mathrm{B}$. With the exception of the data obtained with the blue targets the scores run notably higher than those obtained with the black-on-color configuration. Previous investigators have noted similar results and have discussed the problem of this type of results. On the basis of a simple intensity- or contrast-discrimination theory of visual acuity no difference is predicted since the retinal intensity distributions for the two contrast directions are mathematically complementary (Byram, 1944).

In curve $\mathrm{C} / \mathrm{W}$ are shown the data obtained with colored targets on a white ground. The pattern of the

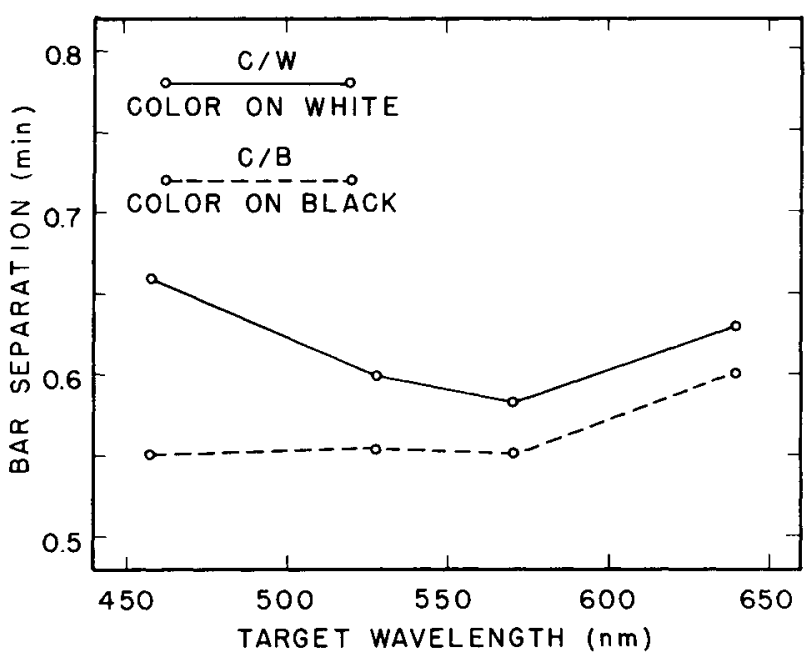

Fi૬. 2. Mean detectable bar separation of $0.5^{\circ} \times 1.1^{\circ}$ colored bars presented on white and black grounds.

scores is the same as that obtained with the black on color configuration with all separation thresholds raised approximately $0.1 \mathrm{~min}$. The relatively low scores clearly suggest that reasonably good visual acuity may be obtained with target ground configurations presenting color contrast only, although high luminance contrast may be a more efficient basis for the discrimination. The results of the experiment suggest, however, that both type and direction of contrast must be considered in an attempt to predict the separation threshold level for a given stimulus ground configuration.

\section{References}

Byram, G. M. The physical and photochemical basis of visual resolving power. Part I. The distribution of illumination in retinal images. J. Opt. Soc. Amer., 1944, 34, 571-591.

Eastman Kodak Company. Influence of color contrast on visual acuity. Report, NDRC contract No. OEmsr-1070, 1944.

Ferree, C. F., \& Rand, G. Visibility of objects as affected by color and composition of light. Part I. With lights of equal luminosity or brightness. Person. J., 1931, 9, 475-492.

Middleton, W. E. K., \& Holmes, M. C. The apparent colors of surfaces of small subtense-a preliminary report. J. Opt. Soc. Amer., $1949,39,582-592$.

Shlaer, S., Smith, E. L., \& Chase, A. M. Visual acuity and illumination in different spectral regions. J. gen. Physiol, 1942, 25, 553-569.

\section{Note}

1. The research reported here was supported by United States Public Health Service Research Grant NB 05088-02 from the National Institutes of Health, Division of Neurological Diseases and Blindness. 